\title{
Engaging Amateurs in the Design, Fabrication, and Assembly of Electronic Devices
}

\author{
David A. Mellis ${ }^{\dagger \dagger}$, Leah Buechley ${ }^{\ddagger}$, Mitchel Resnick ${ }^{\ddagger}$, Björn Hartmann ${ }^{\dagger}$ \\ †: UC Berkeley EECS, $\ddagger$ : MIT Media Lab \\ mellis@berkeley.edu, buechley@gmail.com, mres@media.mit.edu, bjoern@berkeley.edu
}

\begin{abstract}
This paper explores personal fabrication as a means of engaging new audiences in the creation of electronic devices, which play an ever-increasing role in our lives but which most people have little involvement in creating. We describe a six-session workshop in which eight participants made wificonnected devices through the design and fabrication of custom printed circuit boards. We explore the implications of using components and processes analogous to those in commercial products, as opposed to the limited set of higherlevel building blocks found in toolkits for hobbyist electronics. We highlight unique advantages and challenges of a personal fabrication approach. We explore the role of attitudes in identifying a suitable audience for these activities. We discuss insights into commercial electronic products offered by personal fabrication and explore its role in a world of massproduced electronic devices. Finally, we suggest opportunities for future tools and technologies.
\end{abstract}

\section{Author Keywords}

Personal fabrication; DIY; maker culture; CAD/CAM; digital fabrication; Internet of Things; hobby electronics

\section{ACM Classification Keywords}

J.6 Computer-Aided Engineering

\section{INTRODUCTION}

In the 1950's and 60's, it was not unusual for hobbyists to assemble electronic products from kits, like those sold by Heath and other companies. These yielded devices similar to commercial products in both function and appearance. They may not have allowed much scope for customization or creativity, but they allowed individuals to participate in the creation of electronic devices for use in their daily lives. [8] Today, the situation is very different. The sophistication of today's commercial electronic products makes them almost impossible for amateurs to understand or replicate. While recent years have seen the rise of a new generation of hardware startups [15] and the Shanzhai manufacturing ecosystem in China [14], this entrepreneurial manufacturing differs substantially from individual hobbyist practice. As a result, even as elec-

Permission to make digital or hard copies of part or all of this work for personal or classroom use is granted without fee provided that copies are not made or distributed for profit or commercial advantage and that copies bear this notice and the full citation on the first page. Copyrights for third-party components of this work must be honored.

Copyright is held by the owner/author(s).

DIS 2016, June 04-08, 2016, Brisbane, QLD, Australia.

ACM 978-1-4503-4031-1/16/06.

http: //dx.doi.org/10.1145/2901790.2901833

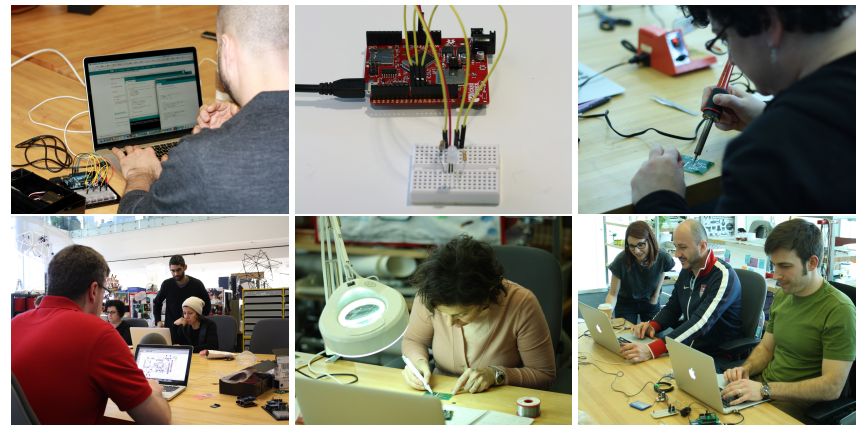

Figure 1. Representative images from each session of the workshop: 1. Arduino, 2. Internet connectivity, 3. example circuit assembly, 4. PCB design, 5. custom circuit assembly, 6. programming and debugging.

tronic devices increasingly dominate our lives, most people are farther and farther removed from understanding or participating in the processes by which those devices are made.

Over the past two decades efforts to engage amateurs in working with electronics have frequently involved toolkits or breadboards. Toolkits (e.g. [2, 7, 13, 22, 32]) provide curated collections of higher-level physical modules which can be combined in a variety of configurations. Breadboards allow for reconfigurable connections between low-level electronic components, provided they come in a compatible $(0.1-$ inch pitch) package. These techniques facilitate rapid tinkering and experimentation by making it easy to combine and recombine electronic components or toolkit modules. Both toolkits and breadboards, however, come with significant limitations. They place restrictions on the components that can be used, limiting the nature and variety of the circuits that can be built. With toolkits, the overhead imposed by wrapping basic components in higher-level building blocks restricts the size and form-factors of the objects built with them. Also, the ease with which toolkit modules or breadboarded components can be connected and disconnected means that they may not be robust enough for long-term use. These limitations make it difficult for toolkits and breadboards to keep pace with the increasing sophistication and optimization of commercial electronic products, making them increasingly less relevant to understanding and participating in the processes by which those products are made.

The increasing capability and accessibility of digital fabrication suggests new possibilities for engaging individuals in the creation of electronic devices. Following Gershenfeld [6], we refer to this personal use of digital fabrication as "personal fabrication." Today, a variety of online ven- 
dors offer on-demand fabrication of custom printed circuit boards (PCBs) in small quantities (e.g. two or three boards). Combined with off-the-shelf electronic components, embedded software, and digitally-fabricated enclosures, these processes can yield complete and robust custom electronic devices. This suggests that fabrication offers possibilities for bringing hobbyist practice closer to the modern world of electronic products. This nature and potential of this approach, however, is not well studied or understood.

Our research investigates the possibilities for engaging amateurs in the personal fabrication of electronic devices. At a basic level, we're interested in to what extent it's even possible to engage non-experts in this process and what factors influence an individual's chance of success. We seek to understand the unique advantages and challenges of a personal fabrication approach relative to alternatives like electronics toolkits and breadboards. We hypothesize that engaging amateurs in the design and fabrication of PCBs will allow them to understand electronic products in ways that go beyond those other approaches. We wonder, however, what role these individuals will envision for PCB design and fabrication in a world full of mass-produced electronic devices. Finally, we hope to learn how, in the future, we can better support new audiences in the design and fabrication of electronic devices.

We explored these topics through a workshop, a format chosen because it allowed us both to support participants through difficult aspects of the process and to closely observe their behavior. The workshop encompassed six five-hour sessions, during which eight participants fabricated custom internetconnected electronic devices. This included selection of electronic components, design of custom printed circuit boards (PCBs), physical assembly (soldering) of the devices, programming, and debugging. As far as possible, we used components, software, and fabrication processes analogous to those found in commercial products - e.g. bare microcontrollers, general purpose PCB design software, and commercial PCB prototyping services. We chose the domain of wificonnected devices because of its relevance to the current interest in the Internet of Things and because it seemed to offer a diversity of applications within a shared technological infrastructure. While we harbored no illusions that workshop participants would be able to compete with the sophistication and complexity of commercial devices, we hoped to bring them closer to the processes by which those products are made. Furthermore, while we recognize the importance of programming in the creation of electronic devices, we choose to focus on other aspects of the process - in part because of the abundance of work on engaging novices in programming (e.g. [20, 23]).

We documented the outcomes of the workshop through observations, surveys, discussions, and interviews. Participants' success in creating their projects suggests that it is indeed possible to engage amateurs in PCB design and fabrication. The workshop reveals unique advantages of personal fabrication - specifically, the robust and flexible circuits it yields and its ability to leverage the power of software in their design. The workshop also highlights some of the specific challenges of a personal fabrication approach - namely project scoping, component selection, and circuit debugging - and suggests strategies for handling them. The experiences of workshop participants suggests that an individual's attitudes are at least as important as their skills in determining the appropriateness of a personal fabrication approach. We show how a personal fabrication approach brings individuals closer to the real world of electronic products, specifically by understanding how they are composed from low-level components, gaining familiarity with the digital processes involved in their design and manufacture, and acquiring a general literacy about electronic devices. While our participants don't envision personal fabrication displacing either mass-produced devices or other forms of electronics prototyping, they do see a place for it - both for replicating successful hobbyist projects and for creating products that wouldn't otherwise exist. Finally, the workshop suggests opportunities for new tools and technologies to engage new audiences in the design and fabrication of electronic circuits, including better PCB design tools, increasing access to automated assembly, and visual programming for embedded systems.

In the next section, we discuss related research. We then describe the workshop, using it to identify advantages and challenges of a personal fabrication approach. We draw on these results for a more general discussion of three themes of importance to personal fabrication. This is followed by our suggestions for future tools and technologies and a brief conclusion.

\section{RELATED WORK}

We discuss three areas of related work: engaging novices with technology, electronics toolkits and prototyping, and the fabrication of interactive devices.

\section{Engaging Novices with Technology}

There's a long history of work on engaging novices in technological making, particularly in the domain of programming. (One prominent early example is Logo [20].) This work has revealed a number of principles beyond simply making the technology itself easier to use. Turkle and Papert [31] argue for the importance of supporting multiple styles of engagement, including hard (top-down) and soft (bottom-up) approaches. Resnick et al. [23] emphasize making systems "more meaningful" and "more social". In our workshop, we similarly sought to identify factors important to engaging new audiences in the personal fabrication of electronic devices.

Many recent discussions view the engagement of novices with technology through the lens of the "maker movement" or "maker culture" [25, 30]. While much of the popular rhetoric surrounding the maker movement tends towards the utopian [29], a number of researchers have offered a more critical perspective $[1,4,5]$. Our work seeks to both engage new audiences in technology production and to explore the broader implications of these technologies.

Other work $([11,19,21])$ has focused specifically on the workshop format. A useful counterpoint is provided by Wakkary et al. [33], who highlight the difficulties of reproducing electronic projects from tutorials. 


\section{Electronics Toolkits and Prototyping}

In the 1990's Resnick and colleagues explored electronics construction kits for children [22, 24]. Toolkits targeting designers, like Phidgets [7] and Calder [13], emerged in the following decade. More recent examples include .NET Gadgeteer [32] and littleBits [2]. These toolkits shield novices from low-level implementation details by encapsulating them in higher-level modules. Greenberg and Fitchett motivate this approach by describing their process of building prototypes before Phidgets:

"We found ourselves immersed in a quagmire of tediousness: selecting and purchasing electrical components and hobby kits, circuit board design, microprocessor programming, wire protocol development, and so on." [7]

While we recognize the value of electronics toolkits, we feel that by avoiding the domains cited by Greenberg and Fitchett, people miss out on valuable opportunities for making and learning. Our workshop explored the value that novices can derive from exploring this "quagmire of tediousness."

Other approaches, like the Arduino platform and the work of Mellis et al. [18], do involve the use of low-level electronic components, whether on breadboards or paper circuits. Neither of these, however, engage novices in the digital design and fabrication of PCBs, a process at the heart of the making of electronic products.

\section{Fabricating Interactive Devices}

There is a variety of related work exploring the use of digital fabrication in the construction of interactive artifacts. One approach is the fabrication of enclosures for .NET Gadgeteer prototypes [32, 34]. Other work, like Sauron [27] and Acoustruments [12], allow for the creation of custom interactive inputs around existing electronic devices (a webcam and smartphone, respectively). Willis et al. [36] 3D-print forms with embedded light guides, enabling the transmission of images and information to and from external circuits.

Some work has looked at new ways of fabricating circuits themselves. Hodges et al. [9] use Microsoft Visio templates to support the layout of inkjet-printed circuits for use with stickers containing electronic components. Midas [28] is a software system for creating capacitive touch inputs. While PCB fabrication itself is not new, we see opportunities for engaging new audiences in the process, particularly as it becomes increasingly accessible through online services. We see new PCB design tools like Fritzing [10] as an important step in this direction but feel there is still much to be learned about how to actually engage people in the PCB design and fabrication process.

Perhaps the closest related work is our previous research [16, 17] in which we created electronic products using digital fabrication. In those workshops, however, participants primarily assembled existing circuit designs or created enclosures for them. In contrast, in the work discussed in this paper, participants engaged in the full process of designing and fabricating a custom electronic circuit, including project definition, component selection, PCB design, and programming.

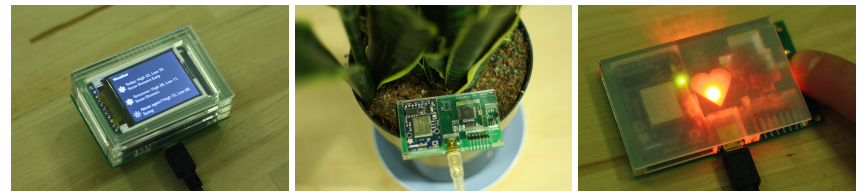

Figure 2. Our example wifi circuits: a display showing the weather (left), a temperature sensor being logged online (center), and an embodied communication device (right).

\section{THE WORKSHOP}

Here we discuss the methodology, participants, technology, structure, and outcomes of our workshop.

\section{Methodology}

We used a workshop format partly as a convenient means of observing and probing novices' processes and perspectives as they engaged with new technologies. The workshop, however, also allowed us to support novices in ways that we suspected would not be well served by existing tools and technologies - as well as to identify opportunities for improving them.

We surveyed participants at the start and end of the workshop and gave them a short questionnaire at the end of each session. We audio-recorded a group discussion at the start and end of the workshop, as well as shorter discussion at the start of individual sessions. Finally, we held (and audio-recorded) one-on-one semi-structured interviews with five of the participants after the conclusion of the workshop.

\section{Participants}

We recruited the workshop participants via fliers in local coffeeshops. We selected eight of the initial fourteen applicants based on their ability to attend all the classes and in an attempt to assemble a diverse set of participants. (See Table 1.) Note that we did not know the level of participants' education when selecting them (and emphasized in our flier that no experience was necessary). We find it interesting that all eight participants had at least a master's degree; this may be due to local demographics in our university environs or to the in-depth nature of the workshop. Participants each paid a fee of $\$ 150$, which covered part of the costs of components and PCB fabrication.

\section{Technology}

We prepared three example devices (Figure 2) to scaffold participants' designs. The examples were intended to showcase a diversity of potential applications of wifi-connected devices. One displayed information from online web services (accessed using Temboo ${ }^{1}$ ) on a small TFT display. Another logged readings from a temperature sensor to an online data store $\left(\right.$ Phant $\left.^{2}\right)$. The third example was a pair of devices for remote communication; touching one caused an LED on the other to glow (and vice-versa).

The examples were intended to demonstrate the design of complete, wifi-connected devices from mass-produced electronic components of the kind that might be found in commercial products. Accordingly, we used a bare, surface-mount ATmega1284P microcontroller as the core of each example.

\footnotetext{
${ }^{1}$ http: //temboo.com/

${ }^{2}$ http: //phant.io/
} 


$\begin{array}{llllllcc}\text { Name } & \text { Age } & \text { Gender } & \begin{array}{l}\text { Race / } \\ \text { Ethnicity }\end{array} & \text { Degree } & \text { Profession } & \begin{array}{c}\text { Programming } \\ \text { Experience }\end{array} & \begin{array}{c}\text { Electronics } \\ \text { Experience }\end{array} \\ \text { Nicole } & 29 & \text { Female } & \text { White } & \text { Masters } & \text { User experience research } & 2 & 2 \\ \text { Tyler } & 30 & \text { Male } & \text { White } & \text { Masters } & \text { Product management / marketing } & 2 & 3 \\ \text { Akash } & 31 & \text { Male } & \text { Asian } & \text { Masters } & \text { Software development } & 5 & 3 \\ \text { Ernesto } & 37 & \text { Male } & \text { Hispanic } & \text { Masters } & \text { Business analysis } & 2 & 1 \\ \text { John } & 42 & \text { Male } & \text { White } & \text { PhD } & \text { Construction building } & 2 & 1 \\ \text { Luisa } & 42 & \text { Female } & \text { White } & \text { Masters } & \text { Architecture and urbanism } & 1 & 1 \\ \text { Nathan } & 44 & \text { Male } & \text { White } & \text { Masters } & \text { Strategy consulting } & 3 & 1 \\ \text { Brooke } & 44 & \text { Female } & \text { White } & \text { Masters } & \text { Environmental management / jewelry } & 2 & 2\end{array}$

Table 1. The workshop participants. (Names are pseudonyms; experience numbers are self-reported on a scale from 1, no experience, to 5, expert.)

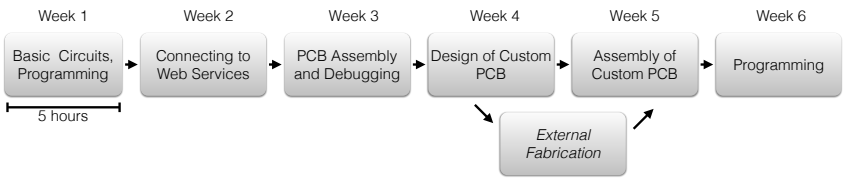

Figure 3. Structure of the six-week workshop.

We could not, however, find a mass-produced wifi module suitable for manual assembly, so we resorted to a breakout board (designed by Adafruit) for the TI CC3000 wifi module. The example PCBs were designed using the Eagle PCB CAD software. Example code was created in the Arduino software development environment.

\section{Structure}

The workshop met from 1 to $6 \mathrm{pm}$ on six consecutive Sundays. Workshop sessions were led by the first author, who is experienced in personal fabrication and teaching electronics workshops. Two other facilitators (with some electronics experience) assisted participants during some of the sessions. Most sessions contained brief facilitator-led demos and lots of time for hands-on experimentation by participants, during which facilitators provided one-on-one support.

The first session introduced basic circuits and code using an Arduino Uno and a breadboard. In the second session, participants learned to connect to web services Temboo and Phant using a Wicked Devices WildFire board (which contains an ATmega1284P and CC3000 wifi module). Up to this point, the workshop was broadly similar to other physical computing or Arduino workshops. During the third session, participants assembled, programmed, and debugged one of the example circuit boards, gaining familiarity with the tools, techniques, and parts they later used with their own designs. Participants designed their PCBs in the fourth workshop session, with seven of the eight starting from our example files. Because our schedule didn't allow participants to prototype their projects on breadboards before designing their PCBs, they relied on individual advice from the workshop facilitators to define the functionality and scope of their circuit boards. After the fourth session, we ordered PCBs for participants' designs from AP Circuits, an online vendor. Because the workshop structure allowed for only a single PCB iteration, we checked their design files for obvious errors before ordering. The PCBs arrived within the week and participants assembled them during the fifth session using components ordered from SparkFun Electronics, Adafruit Industries, and DigiKey. During the last session, participants programmed their

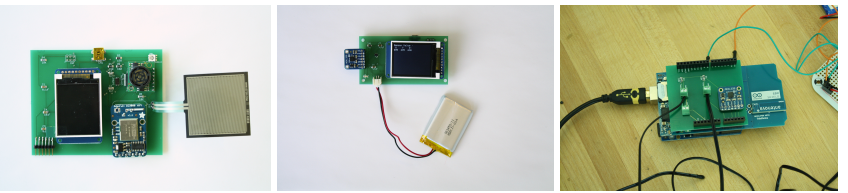

Figure 4. Participant projects: John's snow detection system (left), Nathan's fencing posture detector (center), and Tyler's ocean weather station (right).

boards. Debugging featured heavily in both the fifth and sixth sessions. (See Figure 1 for representative images from each week of the workshop.)

\section{Results}

Table 2 provides an overview of participants' projects, which differed substantially in complexity (i.e. our rough estimate of work they did beyond our provided examples). Tyler ${ }^{3}$ designed his board from scratch, without use of our examples. His project was an Arduino shield intended as the core of an ocean-based weather station. It had thermistors for measuring air and water temperature, accepted power from a solar panel, and transmitted data using the cellular network (via an Arduino GSM shield). Nathan, Akash, John, and Luisa each added multiple sensors and/or actuators to our provided examples, addressing a number of distinct applications areas. Brooke, Ernesto, and Nicole made simpler modifications to our examples, adding only a single sensor or actuator (or, in Brooke's case, two identical RGB LEDs). (See Figure 4 for images of some of the projects.)

In the end, seven of the eight participants successfully assembled, programmed, and debugged their boards (with the exception of one of the two RGB LEDs on Brooke's board). Notably, four of those seven had no experience with electronics prior to the workshop. Four of the participants (Tyler, Nathan, Brooke, and Ernesto) implemented a standalone application for their project, allowing it to function without a computer. Three others (Akash, John, and Luisa) implemented basic examples to test their projects' hardware. Nicole struggled with the soldering, not getting her board to the point where it could be programmed by the end of the workshop.

\section{THE ADVANTAGES AND CHALLENGES OF A PERSONAL FABRICATION APPROACH}

The experiences and reflections of our workshop participants highlight a number of specific advantages and challenges of a personal fabrication approach to the making of electronic devices. We discuss these here, contrasting them with other ap-

\footnotetext{
${ }^{3}$ All names are pseudonyms.
} 


\begin{tabular}{|c|c|c|c|c|c|}
\hline Name & Project & Complexity & Components Added to PCB & HW Status & SW Status \\
\hline Tyler & $\begin{array}{l}\text { Ocean weather } \\
\text { station }\end{array}$ & High & $\begin{array}{l}\text { Thermistor (x2), accelerometer, } \\
\text { solar panel, Arduino shield headers }\end{array}$ & Fully Functional & $\begin{array}{l}\text { Autonomous } \\
\text { application }\end{array}$ \\
\hline Nathan & $\begin{array}{l}\text { Fencing posture } \\
\text { detector }\end{array}$ & Medium & $\begin{array}{l}\text { Accelerometer, LiPo battery, } \\
\text { battery-charging circuit }\end{array}$ & Fully Functional & $\begin{array}{l}\text { Autonomous } \\
\text { application }\end{array}$ \\
\hline Akash & Smart home hub & Medium & $\begin{array}{l}\text { XBee radio, voltage regulator, } \mathrm{RGB} \\
\text { LED }\end{array}$ & Fully Functional & Basic example \\
\hline John & $\begin{array}{l}\text { Snow detection } \\
\text { system }\end{array}$ & Medium & $\begin{array}{l}\text { Ultrasonic range-finder, pressure } \\
\text { sensor, RGB LED }\end{array}$ & Fully Functional & Basic example \\
\hline Luisa & $\begin{array}{l}\text { Migraine } \\
\text { predictor }\end{array}$ & Medium & $\begin{array}{l}\text { Pressure / temperature sensor, } \\
\text { humidity / temperature sensor, RGB } \\
\text { LED }\end{array}$ & Fully Functional & Basic example \\
\hline Ernesto & Energy monitor & Low & Current sensor & Fully Functional & $\begin{array}{l}\text { Autonomous } \\
\text { application }\end{array}$ \\
\hline Brooke & $\begin{array}{l}\text { Email-controlled } \\
\text { LED }\end{array}$ & Low & RGB LED (x2) & Mostly Functional & $\begin{array}{l}\text { Autonomous } \\
\text { application }\end{array}$ \\
\hline Nicole & Unfinished & Low & RGB LED & Incomplete & Not started \\
\hline
\end{tabular}

proaches, like electronic toolkits and breadboards. (While we did not conduct a direct comparison as a part of this work, all the authors have multiple years of experience teaching electronics in various forms, which gives us insight into the characteristics of these other approaches.)

\section{Advantages}

Here we discuss two significant advantages of a PCB fabrication approach: the first regarding its product (the PCB itself) and the second the process by which the PCB is designed.

The product: robust circuits with a diversity of components Our workshop participants found value in the robustness and wide range of components supported by PCB fabrication. As Tyler put it:

"I like the idea of [PCB fabrication] because often times you're just limited to whatever you can buy: what they decided to put on a breakout board at Adafruit, or SparkFun, or Pololu. This kind of opens it up a little more widely.... Being able to just make it all compact and fit neatly, as opposed to I have this one little RadioShack perma-breadboard that I soldered stuff on and then I'm going to have little jumper wires coming over." (Tyler, post-workshop interview)

That is, he appreciates both the ability to drawn on off-theshelf components that haven't been wrapped into higher-level hobbyist modules and the possibility of making a compact and robust project.

These advantages were also visible in the projects themselves (some of which are shown in Figure 4). These are compact and robust, without the chaos of jumper wires found in many breadboard projects. In addition, they draw on a wider range of components than those found in any particular electronics toolkit. For example, neither littleBits nor the .NET Gadgeteer toolkit made by GHI Electronics contain a barometric pressure sensor (used in Luisa's project), a distance sensor / range finger (in John's project), or a LiPo battery charging module (in Nathan's project).

\section{The process: leveraging the power of software}

Our participants also valued the scaffolding provided by designing their circuits in software, particularly the ease of building on existing examples and the ability to leverage automated processes. Akash valued the ability to build on existing design files:

"Yeah, I mean, the designing the boards seemed a lot easier than I anticipated, I guess because we started with some templates and just made some modifications." (Akash, closing discussion)

This is stark contrast to work with breadboard prototypes, where building on someone else's circuit design involves first recreating it on your own breadboard, a manual and errorprone process. With a personal fabrication approach, you can start by just making changes to someone else's file. Furthermore, the resulting files can be shared for others to build on or fabricate.

Software can also automate what would otherwise be tedious manual processes, an ability valued by participants:

"Eagle was nice. I could definitely see how you could have to spend a lot of time needing to become a real pro at it but the auto-router was magical. It was cool watching all the wires go bo-bo-bo-bop." (Tyler, closing discussion)

"Yeah, laying out traces was way more easy than I thought it would be. I had no idea that there was such a thing as the auto-router." (Brooke, closing discussion)

The auto-router provides a hint of the way in which the power and flexibility of software could be leveraged to support novices in the circuit design process, a topic we return to in "Opportunities for Future Tools and Technologies" below.

\section{Challenges}

Here we discuss three specific challenges that emerged from the workshop: scoping projects, component selection and integration, and debugging circuits of low-level components. 


\section{Scoping Projects}

The many up-front decisions required by a personal fabrication approach made it particularly difficult for our participants to define and scope their projects. Nathan nicely captured his difficulty with the process:

\begin{abstract}
"My problem was I didn't have a problem I wanted to solve and so I was like I'm just going to try to eyeball something and come up with something out of the air. And, [first author's name] and I bounced a few ideas back and forth... Part of the issue was scale. I thought of a few projects that I would do and I was thinking 'yeah that would be really interesting and would take two years to put together so I'm going to put that one aside for now.' And then others, were like 'oh, yeah, that's a great idea and I can go buy that at Walmart today for five dollars so I'm not going to do that either."' (Nathan, closing discussion)
\end{abstract}

While many of the considerations Nathan mentions, such as scale, novelty, and expertise, are also relevant to work with toolkits and breadboards, the iterative nature of those approaches facilitates the process of project scoping. In contrast, the slow iteration cycles found in a personal fabrication approach require making more decisions about projects upfront, without the increased experience and insight enabled by more frequent project iterations.

Our experience in the workshop suggests some potential solutions to this challenge. One option is to work with people that have already come up with a feasible project idea. This was the case with Tyler; it enabled him to focus on implementing his idea through personal fabrication. Another possibility is to work with people that are content to go through the PCB design process without a specific project goal. This was the case with Brooke and Ernesto, both of whom chose to simply add one or two basic components to the example PCB designs. If neither of these cases apply, Nathan's experience suggests that it will be necessary to dedicate significant time and individual support to the project scoping process.

\section{Component selection and integration}

The wide range of low-level components that can be used with a custom PCB increases the challenge of selecting the right ones and integrating them into a functional circuit. Doing so requires taking account of multiple factors, which are nicely captured in Tyler's account of selecting a temperature sensor for his project:

"What do I want to do with this? I want to measure temperature. Go on Adafruit, go on SparkFun, all the different ways of measuring temperature. Do I use a thermocouple? Do I use a thermistor? Read a bunch of reviews... read the tutorials. Okay, thermistor it is; it's waterproof, it's simple. I don't have to do any weird calibration or logic shifting." (post-workshop interview)

Here, Tyler is weighing a number of different factors: component availability, ease-of-interfacing (along multiple dimensions), and robustness. The sophistication of the process he describes was a challenge for many of our participants. Fur- thermore, even once they selected a component, they often struggled to understand how to use it. As Luisa put it:

"What I found challenging was to understand what are the components that have to be paired with the basic components that you are buying. Like how many resistors, how many capacitors, where is the crystal, why one and not two or not three.... The reasoning behind the selection of all these things beyond the sensors that we buy directly to Adafruit." (closing discussion)

That is, while Luisa was comfortable with the high-level idea of selecting a sensor to accomplish a particular function, she struggled to understand the details necessary to make use of those sensors. We also observed participants struggling to coordinate information from diverse sources, such as Eagle part libraries, Arduino software libraries, component product listings and datasheets, and online tutorials. In contrast, an electronics toolkit will typically encapsulate the information required to work with individual components, avoiding many of these difficulties. Our workshop, however, also suggests that wrestling with these challenges provides people with valuable learning experiences, a point we return to in the discussion.

\section{Debugging Circuits of Low-Level Components}

For our participants, debugging circuits boards composed of individual low-level components was a challenge. Nathan expressed his frustration with the process:

"My biggest stumbling block was not even debugging the code but just debugging my board.... Honestly my first reaction when I couldn't get this to upload was 'screw it, I've got another one, I'll start over' because I don't where to begin, I don't know where to step into the process." (closing discussion)

That is, Nathan had difficulty formulating hypotheses as to what might be wrong with his circuit, a challenge shared by other participants. With personal fabrication, this difficulty is compounded because of the necessity of debugging individual components and connections. In contrast, when working with an electronics toolkit, individual modules typically function, meaning that debugging operates at a higher level of abstraction, making it easier to postulate what might be wrong. We see opportunities for new tools and technologies to help with the debugging process, a topic we discuss in "Opportunities for Future Tools and Technologies" below.

\section{DISCUSSION}

Here, we discuss three themes relevant to engaging amateurs in personal fabrication: the role of existing attitudes, not just skills, in determining the appropriateness of a personal fabrication approach; the ways in which personal fabrication brings individuals closer to world of commercial electronic products; and the role of personal fabrication in a world of mass-produced devices.

\section{The Importance of Attitudes, Not Just Skills}

Here, we discuss the importance of attitudes, not just skills, in determining the appropriateness of a personal fabrication approach for engaging an individual in the making of electronics. We look at the relationship between the specific 
characteristics of PCB design and fabrication and issues of self-efficacy, motivation and context, and alignment with individual goals. These themes emerged from an iterative analysis of the experience of the individual workshop participants, informed by discussions among the authors and other researchers.

\section{The Role of Self-Efficacy}

The contrasting experience of two of our workshop participants, Nathan and Nicole, highlights the importance of selfefficacy in overcoming the particular challenges of a personal fabrication approach to electronics. This is a small data-point in a larger discussion about the role of self-efficacy in computer science and other technological activities (e.g. [3, 26, $35])$. In this instance, the importance of self-efficacy was illustrated by Nathan and Nicole's attempts at soldering and debugging their PCBs. Both participants entered with the workshop with more-or-less comparable skills but reacted in very different ways to the challenges they encountered.

When Nathan ran into difficulties debugging his circuit board, he was initially lost but persevered, drawing on help from the workshop facilitators to get his board working. This perseverance was reflective of his confidence in his ability to solve problems, as expressed earlier in the workshop:

"It looks like I have enough of the tools that I can, you know, if there's some little thing that I don't understand I can figure it out, or just drive over it really...." (third session)

That is, Nathan had the confidence to continue working in the face of difficulty, although he sometimes required support to do so. Nicole, in contrast, gave up when she ran into trouble soldering her PCB, despite help and encouragement from the facilitators. (While impossible to confirm, we suspect that Nicole could have successfully assembled her board had she persisted in her efforts.) Nicole also skipped the last workshop session out a desire not to burden the rest of the participants with her difficulties. Later, Nicole expressed a lack of confidence in her abilities:

"Nothing I make ever works.... I can try really hard to be exact about things but there's always a problem.... I feel like y'all can do something and then- I don't know, it just doesn't ever work out for me.” (post-workshop interview)

Although some of the self-doubt expressed in this quote may have been a post-hoc rationalization, it seems likely to have played at least some part in her lack of perseverance.

We hypothesize that self-efficacy plays an especially important role in personal fabrication because of the large overhead required to get anything to work at all. This provides fewer opportunities for success as compared to the tinkering possible with toolkits and breadboards; this lack of positive reinforcement may discourage those with less confidence in their own abilities.

The Value of a Dedicated Context

Our participants benefited from the structure and motivation they derived from our workshop context. Tyler's experience exemplifies the importance of this context in supporting people's engagement with personal fabrication. As evidenced by the complexity of his project and his ability to work largely without assistance in the workshop, Tyler already had many of the technical skills necessary to design and fabricate a PCB. Still, he had struggled with previous attempts at electronics. As he put it:

"There was no one around me who was doing it, so there was no one I could talk about it with... I know all this stuff is online but there's so much online that it's almost overwhelming. It just seemed like you had to be more of an expert to even get started. That might not have been true. And actually with experience in the class, I know now that that wasn't true. But that was my perception of it." (post-workshop interview)

That is, Tyler had interpreted his difficulties as a lack of the necessary expertise, when, in fact, they seem to really have been about the lack of a supportive context. While the internet offered an abundance of information, it wasn't until our workshop that Tyler found the focus and structure he needed to exercise his abilities.

Other participants, too, appeared to benefit from the workshop structure. Some, for instance, borrowed workshop materials with the intention of working at home but returned the next week without having found the time to do so. On the other hand, they were dedicated to the workshop itself, with only two participants missing even a single session (one because he was out of the country).

In addition, the in-person, one-on-one support enabled by a workshop context was crucial to helping participants overcome the challenges of a personal fabrication approach. Project scoping, component selection and integration, and PCB debugging all required a lot of back-and-forth between the facilitators and workshop participants, a process made much easier by in-person conversation. Furthermore, helping participants debug their PCBs would have been quite difficult without physical access to the boards.

These issues highlight the importance of a dedicated structure and social context in motivating novices to invest time and effort in developing new knowledge and skills as well as in supporting them through the process. Of course, holding an in-person workshop isn't always possible, so it's interesting to consider other means of providing this type of support, a question we return to in "Opportunities for Future Tools and Technologies" below.

\section{The Importance of Alignment with Individual Goals}

Here, we contrast the experiences of Ernesto and Luisa as a means of highlighting the importance of aligning personal fabrication activities with an individual's personal goals. Ernesto explained his motivations: "I really want[ed] to be able to see the whole process from the beginning to the end so I picked a very simple project" (closing discussion). This aligned well with our workshop structure, which offered an overview of the many different processes involved in designing and fabricating an electronic device. In contrast, Luisa entered the workshop with an interest in a specific applica- 
tion domain (sensors and the built environment) of relevance to her profession (architecture and urbanism). While she was successful at and enjoyed the process of designing and assembling her PCB - executing a more complex project that Ernesto - she would have preferred a different focus:

"I loved Eagle but if I would remove something maybe that would be the part that I would remove." (closing discussion)

That is, despite Luisa's skill at and enjoyment of PCB design, our workshop's focus on these processes was not a good fit for her personal interests and goals. These may have aligned better with the qualities of electronics toolkits and breadboard prototyping, which would have allowed for more experimentation within the application domains of interest to Luisa. This is a useful reminder that personal fabrication isn't always the most appropriate approach, even for those with the potential to do it successfully.

\section{Approaching the Real World of Electronic Products}

The workshop suggests that a personal fabrication approach offers important insights into the way that electronic products are made - insights not revealed by other approaches like electronics toolkits. While they don't include every aspect of the product design and manufacturing process, we feel they offer important steps in that direction. Here, we discuss two of these insights: the composition of electronic devices from low-level components and the role of digital design and fabrication processes.

\section{Compositions of low-level components}

By taking a personal fabrication approach to the making of electronic devices, our participants gained an understanding of the way in which commercial electronic products are composed from commercial, off-the-shelf electronic components, a perspective often obscured by the abstractions offered by electronics toolkits. As Akash put it (before we started to work with bare microcontrollers):

"One thing that I still don't understand, that I'd like to learn more about... this Arduino board is still kind like a black box to me." (session three)

That is, the toolkit substitutes one black box (an electronic product) with another (a toolkit module), both of which are at a similar level of abstraction (i.e. multiple electronic components mounted on a PCB). Furthermore, an Arduino board is a hobbyist module that one wouldn't expect to see inside of a commercial device. In contrast, a personal fabrication approach allows novices to go down a level of abstraction and see how electronic circuits are composed from individual components of the sort found in commercial devices. As John put it:

"Yeah, I think at some systems level or sub-systems level I've a better understanding now of how things work. Maybe down at the minute level ... do I still understand how this sensor works? I probably don't." (closing discussion)

This understanding of the way in which devices are composed from individual components is similar to the level of abstrac- tion involved in creating many commercial electronic products, which also rely on the encapsulation of electronics theory provided by off-the-shelf sensors and other components. That is, just as a professional engineer can make use of an existing sensor or microcontroller without necessarily knowing the theory governing its operation, so, too, John now has an understanding of the way that electronic products are composed from these individual components.

For Brooke, there was even value in the fact that our workshop components came in commercial packaging rather than as a kit of parts:

"I really appreciate that we didn't use a kit to make things but did our own and used components we had to fish out of bags. Felt more real and less like being led around." (post-workshop survey)

This simple step - taking individual components from their industrial packaging - gave Brooke a sense of participating in the real world of electronic products rather than the limited scope of a pre-packaged kit. For us, this highlights the potential for personal fabrication to transform hobbyist electronics - from tinkering with a limited set of toolkit modules to engaging with the general domain of electronic components.

\section{Digital design and fabrication processes}

While personal fabrication uses different manufacturing processes than mass production, it similarly involves the translation of digital designs into physical artifacts, a paradigm which is essential to understanding how commercial devices are made. At the end of the workshop, Ernesto described his understanding of this digital design and manufacturing process:

"How all the pieces interact, Eagle - you got two different files, you send them to Canada. They send you something back. You get sensors from Adafruit. You have SparkFun. Like how all those pieces interact together just to be able to control a physical device from code." (closing discussion)

While this is clearly a long way from knowing all the details involved in mass-produced an electronic device, it represents a general overview of that process, one which stems directly from Ernesto's experience in designing and fabricating a PCB.

Brooke gained a better understanding of the PCB design process in particular:

"I think that the Eagle part was really useful because I didn't know ... how hardware designers pull everything together. I didn't know about libraries where you can just grab parts and it definitely demystified that part." (closing discussion)

That is, she now understands some of the concrete steps involved in what was previously a generally-mysterious process of electronic product design. Again, while this is clearly a long way from the full knowledge required to design a commercial electronic product, it expresses an significant demystification of that process. 
Together, these quotes illustrate the overview of digital design and manufacturing afforded by the personal fabrication of an electronic device. As with an appreciation for the use of low-level electronic components, this insight is fundamental in fostering a shift from the world of pre-made toolkit modules to the commercial world of electronic products.

\section{Finding a Role for Personal Fabrication}

Participants emerged from the workshop with an informed and nuanced understanding of the value and role of a personal fabrication approach to the making of electronic products. On the one hand, they recognized that it was not likely to replace mass-production as the means by which most electronic devices are made. On the other hand, they felt that it could have a role to play in their own personal practices - and that it offers opportunities to create products that aren't currently provided by industry.

Participants were skeptical that personal fabrication would become someone's primary means of creating the devices in their lives. As Tyler put it:

"I don't think I'm going to be building the next iPhone or making my own- like some people learn how to make their own beer, and they never drink any beer cause they're just 'I'm going to drink my own beer cause it's the best and I'm the best'- I don't think that's going to happen with this." (Tyler, closing discussion)

That is, Tyler doesn't envision personal fabrication replacing mass-production for most electronic devices, an opinion shared by other workshop participants - and one which we generally agree with. Given this, the real question for us is what role personal fabrication has to play in society.

Comments from our participants suggest they see the possibility of incorporating PCB design and fabrication into their own practice:

"I think though if I had a project that needed a lot of boards like the same boards over and over, I would get it to this stage.... I probably wouldn't pay $\$ 80$ for a board that ... I knew that I was only going to need one of in my whole life. But if I had a reason to, I would definitely go down that route." (Brooke, closing discussion)

“The Eagle was really interesting to learn and I'm sort of like, you know for a hundred bucks if I think of something I want to do, maybe I go down that road. Cause it was kind of interesting and if anything for me it's an interesting conversation piece." (Nathan, closing discussion)

"But, yeah, I mean, if I'm making a one-off thing and none of my friends are ever going to use it, I'll probablyI might just make it but I wouldn't do a PCB, I'd do it on a solderable breadboard. If I was making something and a bunch of people around me showed interest, yeah, maybe I'd go ahead and whip up Eagle and start doing some things." (Tyler, post-workshop interview)

That is, while a personal fabrication approach might not be the only (or even the primary) way that they will work with electronics in the future, it's now a part of their repertoire that they can draw on when appropriate. Specifically, personal fabrication offers these participants a way to replicate, in robust form, a successful design and share it with others. This is a quality that is difficult to achieve with breadboard and electronic toolkit prototypes. We find it especially encouraging that, having seen the work involved in designing and fabricating a PCB, these participants still feel it is something that they might engage in for future projects.

Furthermore, participants saw personal fabrication as a process that could allow for the creation of products that wouldn't otherwise exist:

"Some of the stuff just isn't out there, like for swimming there's really not that great electronics.... I've been thinking about... what if I could have a heading indicator on my goggles? That's just a thing that no one makes." (Tyler, post-workshop interview)

"If I would have been in Colombia, I would have just jumped straight into setting a business... because I see a huge market there, a necessity for these products. It wouldn't have been difficult at all to start a business there." (post-workshop interview)

These participants see the potential for devices that companies don't make and view personal fabrication as an opportunity for individuals to get involved in the creation of those devices. Together with the previous comments, this suggests that our participants do see a clear role for personal fabrication of electronic devices, both in individual hobbyist practice and as a means of supporting the creation of new electronic products. While toolkits and breadboards are great for novices tinkering with electronics, personal fabrication offers a path for the amateur to create robust, repeatable prototypes of electronic devices. This extends the real-world applications of hobbyist electronics, echoing the paths from experimentation to use found in many other hobbies - cooking, wood-working, knitting, and more.

\section{OPPORTUNITIES FOR FUTURE TOOLS AND TECHNOLO- GIES}

Participants' experience in the workshop revealed multiple opportunities for future tools and technologies to support individuals in the creation of electronic products. These tools and technologies seem likely to increase in importance as the range of digital fabrication technologies accessible to hobbyists continues to expand.

\section{Better Abstractions in Circuit Design Software}

As discussed in "Advantages of a PCB Fabrication Approach" above, Eagle's auto-router provides one example of leveraging the power of software (and embedded domain expertise) to support novice design of circuits. Our experience with the workshop suggests other kinds of support that would be helpful to embed in future PCB design tools.

Currently, the PCB design tools available to hobbyists (like Eagle) provide little or no support for creating abstractions of multiple components. (Eagle doesn't even provide very good support for copying and pasting between files.) A PCB 
design tool with better support for such abstractions could help users focus on the higher-level functionality they want to include in their circuits without needing to worry about each individual component. Providing higher-level building blocks in software rather than as hardware modules would offer a number of advantages: they could draw on a wider range of electronic components, they could be easily shared online, and they could be tightly integrated onto a single PCB.

Another powerful resource would be encoding more electronics knowledge into PCB design tools. For example, supporting users in connecting components to microcontroller pins with specialized functionality (e.g. analog inputs, SPI, or I2C communication) necessary to work with those components. Also useful would be helping them to translate between different vocabularies, such as the name of a communications bus (e.g. SPI) and the names of its individual signals (MISO, MOSI, SCK). Both of these issues tripped up many of the participants in our workshop. Embedding this type of knowledge into PCB design software would also make it possible to guide the user through the process of debugging their fabricated circuit boards. For instance, the software could suggest specific solder joints or connections to test based on the observed circuit behavior or suggest the minimal set of components required to get a particular portion of the board working.

\section{Increasing Access to Automated Circuit Assembly}

Increased hobbyist access to automated low-volume circuit assembly would increase the range of components available for and reduce the manual labor involved in creating custom circuits. It would also move much of the onus for testing and debugging to the vendor, who is likely better and more efficient at this task. This would increase the importance of the digital design process, further distinguishing a personal fabrication approach from breadboards and toolkits and increasing its potential sophistication and importance. There are some promising initiatives in this area, like the PCB assembly service from Seeed Studio ${ }^{4}$ and Octopart's common parts library ${ }^{5}$.

\section{Leveraging Lessons on Novice Coding}

Given the centrality of programming in the creation of electronic products, we see important opportunities to translate lessons on supporting novice programming from other domains (e.g. visual programming interfaces). Specifically, the workshop demonstrated a need for tools and interfaces that simplify embedded development while still allowing for the use of a wide range of electronic components, which requires an open-endedness and flexibility sometimes missing from novice coding environments.

Distributed Social Contexts for Technology Engagement While not necessarily specific to our personal fabrication approach, we want to re-iterate the value of the in-person support and social context offered by a workshop (in contrast to purely digital scaffolding). One advantage of this format is supporting participants in complex and subjective parts of

\footnotetext{
${ }^{4}$ http: //www.seeedstudio.com/

${ }^{5}$ https://octopart.com/common-parts-library/
}

the design and fabrication process, like project scoping and debugging (discussed in "Challenges of a PCB Fabrication Approach" above). Another is providing a setting that motivates people's work and allows them to focus on a particular domain.

While we feel that in-person workshops are an ideal means of providing this type of social context and support, we recognize that they don't necessarily scale. An interesting question for future exploration is how to create distributed, online environments that can provide structure and support for technology making. While there are many initiatives in this area ${ }^{6}$, we feel there's still interesting work to be done in figuring out how to shape and scale these offerings.

\section{CONCLUSION}

We presented an investigation into engaging amateurs in the design, fabrication, and assembly of electronic devices - a six-session workshop in which eight participants created wificonnected devices. We've shown that it is possible to engage non-experts in the personal fabrication of electronic devices and that doing so brings them closer to the real world of commercial electronic products, as compared with other approaches like toolkits or breadboards. Furthermore, we've demonstrated that amateurs do indeed see a role for personal fabrication, even in a world full of mass-produced electronic devices. On the other hand, there are many challenges involved in a personal fabrication approach; we've identified some important ones and suggested opportunities for addressing them. We've also highlighted some of the many considerations involved in determining the appropriateness of a personal fabrication approach for different audiences. In short, we've shown that personal fabrication allows amateurs to create robust, repeatable devices, extending the scope of hobbyist electronics and its potential for real-world applications.

\section{ACKNOWLEDGMENTS}

This work was supported in part by the TerraSwarm Research Center, one of six centers supported by the STARnet phase of the Focus Center Research Program (FCRP) a Semiconductor Research Corporation program sponsored by MARCO and DARPA. Thanks to all the workshop participants and to Alexis Hope and Santiago Alfaro for their help facilitating the workshop!

\footnotetext{
${ }^{6}$ See, for example, EE40LX from UC Berkeley on edX (https : // www.edx.org/course/electronic-interfaces-bridgingphysical-uc-berkeleyx-ee $401 \mathrm{x}-0$ ), the Stanford HCI course on Coursera (https://class.coursera.org/hci/lecture), and Learning Creative Learning from the MIT Media Lab (http://learn.media.mit.edu/lcl/)
} 


\section{REFERENCES}

1. Morgan G. Ames, Jeffrey Bardzell, Shaowen Bardzell, Silvia Lindtner, David A. Mellis, and Daniela K. Rosner. 2014. Making Cultures: Empowerment, Participation, and Democracy - or Not?. In Proceedings of the Extended Abstracts of the 32Nd Annual ACM Conference on Human Factors in Computing Systems (CHI EA '14). ACM, New York, NY, USA, 1087-1092. DOI : http://dx.doi.org/10.1145/2559206.2579405

2. Ayah Bdeir. 2009. Electronics As Material: LittleBits. In Proceedings of the 3rd International Conference on Tangible and Embedded Interaction (TEI '09). ACM, New York, NY, USA, 397-400. DOI : http://dx.doi.org/10.1145/1517664.1517743

3. Laura Beckwith, Cory Kissinger, Margaret Burnett, Susan Wiedenbeck, Joseph Lawrance, Alan Blackwell, and Curtis Cook. 2006. Tinkering and Gender in End-user Programmers' Debugging. In Proceedings of the SIGCHI Conference on Human Factors in Computing Systems (CHI '06). ACM, New York, NY, USA, 231-240. DOI : http: //dx.doi.org/10.1145/1124772.1124808

4. Leah Buechley. 2014. Thinking About Making. Talk, Eyeo Festival. (2014).

5. Sarah Fox, Rachel Rose Ulgado, and Daniela Rosner. 2015. Hacking Culture, Not Devices: Access and Recognition in Feminist Hackerspaces. In Proceedings of the 18th ACM Conference on Computer Supported Cooperative Work \&\#38; Social Computing (CSCW '15). ACM, New York, NY, USA, 56-68. DOI : http: //dx.doi.org/10.1145/2675133.2675223

6. Neil Gershenfeld. 2007. Fab: The Coming Revolution on Your Desktop-from Personal Computers to Personal Fabrication. Basic Books, Inc., New York, NY, USA.

7. Saul Greenberg and Chester Fitchett. 2001. Phidgets: Easy Development of Physical Interfaces Through Physical Widgets. In Proceedings of the 14th Annual ACM Symposium on User Interface Software and Technology (UIST '01). ACM, New York, NY, USA, 209-218. DOI :

http: //dx.doi.org/10.1145/502348.502388

8. Kristen Haring. 2007. Ham Radio's Technical Culture. MIT Press, Cambridge, MA, USA.

9. Steve Hodges, Nicolas Villar, Nicholas Chen, Tushar Chugh, Jie Qi, Diana Nowacka, and Yoshihiro Kawahara. 2014. Circuit Stickers: Peel-and-stick Construction of Interactive Electronic Prototypes. In Proceedings of the 32Nd Annual ACM Conference on Human Factors in Computing Systems (CHI'14). ACM, New York, NY, USA, 1743-1746. DOI : http: //dx.doi.org/10.1145/2556288.2557150

10. André Knörig, Reto Wettach, and Jonathan Cohen. 2009. Fritzing: A Tool for Advancing Electronic Prototyping for Designers. In Proceedings of the 3rd International Conference on Tangible and Embedded Interaction (TEI '09). ACM, New York, NY, USA, 351-358. DOI : http: //dx.doi.org/10.1145/1517664.1517735
11. Stacey Kuznetsov, George Noel Davis, Eric Paulos, Mark D. Gross, and Jian Chiu Cheung. 2011. Red Balloon, Green Balloon, Sensors in the Sky. In Proceedings of the 13th International Conference on Ubiquitous Computing (UbiComp '11). ACM, New York, NY, USA, 237-246. DOI : http: //dx.doi.org/10.1145/2030112.2030145

12. Gierad Laput, Eric Brockmeyer, Scott E. Hudson, and Chris Harrison. 2015. Acoustruments: Passive, Acoustically-Driven, Interactive Controls for Handheld Devices. In Proceedings of the 33rd Annual ACM Conference on Human Factors in Computing Systems (CHI'15). ACM, New York, NY, USA, 2161-2170. DOI : http: //dx.doi.org/10.1145/2702123.2702414

13. Johnny C. Lee, Daniel Avrahami, Scott E. Hudson, Jodi Forlizzi, Paul H. Dietz, and Darren Leigh. 2004. The Calder Toolkit: Wired and Wireless Components for Rapidly Prototyping Interactive Devices. In Proceedings of the 5th Conference on Designing Interactive Systems: Processes, Practices, Methods, and Techniques (DIS '04). ACM, New York, NY, USA, 167-175. DOI : http: //dx.doi.org/10.1145/1013115.1013139

14. Silvia Lindtner, Anna Greenspan, and David Li. 2015. Designed in Shenzhen: Shanzhai Manufacturing and Maker Entrepreneurs. Aarhus Series on Human Centered Computing 1, 1 (2015), 12. DOI: http://dx.doi.org/10.7146/aahcc.v1i1.21265

15. Silvia Lindtner, Garnet D. Hertz, and Paul Dourish. 2014. Emerging Sites of HCI Innovation: Hackerspaces, Hardware Startups \&\#38; Incubators. In Proceedings of the SIGCHI Conference on Human Factors in Computing Systems (CHI'14). ACM, New York, NY, USA, 439-448. DOI : http: //dx.doi.org/10.1145/2556288.2557132

16. David A. Mellis and Leah Buechley. 2012. Case Studies in the Personal Fabrication of Electronic Products. In Proceedings of the Designing Interactive Systems Conference (DIS '12). ACM, New York, NY, USA, 268-277. DOI :

http: //dx.doi.org/10.1145/2317956.2317998

17. David A. Mellis and Leah Buechley. 2014.

Do-it-yourself Cellphones: An Investigation into the Possibilities and Limits of High-tech Diy. In Proceedings of the SIGCHI Conference on Human Factors in Computing Systems (CHI '14). ACM, New York, NY, USA, 1723-1732. DOI : http: //dx.doi.org/10.1145/2556288.2557309

18. David A. Mellis, Sam Jacoby, Leah Buechley, Hannah Perner-Wilson, and Jie Qi. 2013. Microcontrollers As Material: Crafting Circuits with Paper, Conductive Ink, Electronic Components, and an "Untoolkit". In Proceedings of the 7th International Conference on Tangible, Embedded and Embodied Interaction (TEI '13). ACM, New York, NY, USA, 83-90. DOI : http://dx.doi.org/10.1145/2460625.2460638 
19. Katherine Moriwaki and Jonah Brucker-Cohen. 2006. Lessons from the Scrapyard: Creative Uses of Found Materials Within a Workshop Setting. AI Soc. 20, 4 (Aug. 2006), 506-525. DOI: http: //dx.doi.org/10.1007/s00146-006-0036-7

20. Seymour Papert. 1980. Mindstorms: Children, Computers, and Powerful Ideas. Basic Books, Inc., New York, NY, USA.

21. Matt Ratto. 2011. Critical Making: Conceptual and Material Studies in Technology and Social Life. The Information Society 27, 4 (July 2011), 252-260. DOI : http: //dx.doi.org/10.1080/01972243.2011.583819

22. Mitchel Resnick. 1993. Behavior Construction Kits. Commun. ACM 36, 7 (July 1993), 64-71. DOI : http: //dx.doi.org/10.1145/159544.159593

23. Mitchel Resnick, John Maloney, Andrés Monroy-Hernández, Natalie Rusk, Evelyn Eastmond, Karen Brennan, Amon Millner, Eric Rosenbaum, Jay Silver, Brian Silverman, and Yasmin Kafai. 2009. Scratch: Programming for All. Commun. ACM 52, 11 (Nov. 2009), 60-67. DOI : http: //dx.doi.org/10.1145/1592761.1592779

24. Mitchel Resnick, Fred Martin, Robert Berg, Rick Borovoy, Vanessa Colella, Kwin Kramer, and Brian Silverman. 1998. Digital Manipulatives: New Toys to Think with. In Proceedings of the SIGCHI Conference on Human Factors in Computing Systems (CHI '98). ACM Press/Addison-Wesley Publishing Co., New York, NY, USA, 281-287. DOI :

http: //dx.doi.org/10.1145/274644.274684

25. David Roedl, Shaowen Bardzell, and Jeffrey Bardzell. 2015. Sustainable Making? Balancing Optimism and Criticism in HCI Discourse. ACM Trans. Comput.-Hum. Interact. 22, 3, Article 15 (June 2015), 27 pages. DOI : http: //dx.doi.org/10.1145/2699742

26. Mary Beth Rosson, John M Carroll, and Hansa Sinha. 2011. Orientation of undergraduates toward careers in the computer and information sciences: Gender, self-efficacy and social support. ACM Transactions on Computing Education (TOCE) 11, 3 (2011), 14.

27. Valkyrie Savage, Colin Chang, and Björn Hartmann. 2013. Sauron: Embedded Single-camera Sensing of Printed Physical User Interfaces. In Proceedings of the 26th Annual ACM Symposium on User Interface Software and Technology (UIST'13). ACM, New York, NY, USA, 447-456. DOI :

http: //dx.doi.org/10.1145/2501988.2501992

28. Valkyrie Savage, Xiaohan Zhang, and Björn Hartmann. 2012. Midas: Fabricating Custom Capacitive Touch Sensors to Prototype Interactive Objects. In Proceedings of the 25th Annual ACM Symposium on User Interface Software and Technology (UIST'12). ACM, New York, NY, USA, 579-588. DOI :

http: //dx.doi.org/10.1145/2380116.2380189
29. Susan Currie Sivek. 2011. We Need a Showing of All Hands: Technological Utopianism in MAKE Magazine. Journal of Communication Inquiry (2011). DOI : http: //dx.doi.org/10.1177/0196859911410317

30. Joshua G. Tanenbaum, Amanda M. Williams, Audrey Desjardins, and Karen Tanenbaum. 2013. Democratizing Technology: Pleasure, Utility and Expressiveness in DIY and Maker Practice. In Proceedings of the SIGCHI Conference on Human Factors in Computing Systems (CHI'13). ACM, New York, NY, USA, 2603-2612. DOI : http://dx.doi.org/10.1145/2470654.2481360

31. Sherry Turkle and Seymour Papert. 1991. Epistemological Pluralism and the Revaluation of the Concrete. In Constructionism, Idit Harel and Seymour Papert (Eds.). Ablex Publishing Corporation.

http: //papert.org/articles/

Epistemologicalpluralism.html

32. Nicolas Villar, James Scott, Steve Hodges, Kerry Hammil, and Colin Miller. 2012. .NET Gadgeteer: A Platform for Custom Devices. In Proceedings of the 10th International Conference on Pervasive Computing (Pervasive'12). Springer-Verlag, Berlin, Heidelberg, 216-233. DOI :

http: //dx.doi.org/10.1007/978-3-642-31205-2_14

33. Ron Wakkary, Markus Lorenz Schilling, Matthew A. Dalton, Sabrina Hauser, Audrey Desjardins, Xiao Zhang, and Henry W.J. Lin. 2015. Tutorial Authorship and Hybrid Designers: The Joy (and Frustration) of DIY Tutorials. In Proceedings of the 33rd Annual ACM Conference on Human Factors in Computing Systems (CHI'15). ACM, New York, NY, USA, 609-618. DOI : http: //dx.doi.org/10.1145/2702123.2702550

34. Christian Weichel, Manfred Lau, and Hans Gellersen. 2013. Enclosed: A Component-centric Interface for Designing Prototype Enclosures. In Proceedings of the 7th International Conference on Tangible, Embedded and Embodied Interaction (TEI'13). ACM, New York, NY, USA, 215-218. DOI :

http://dx.doi.org/10.1145/2460625.2460659

35. Susan Wiedenbeck. 2005. Factors Affecting the Success of Non-majors in Learning to Program. In Proceedings of the First International Workshop on Computing Education Research (ICER '05). ACM, New York, NY, USA, 13-24. DOI :

http: //dx.doi.org/10.1145/1089786.1089788

36. Karl Willis, Eric Brockmeyer, Scott Hudson, and Ivan Poupyrev. 2012. Printed Optics: 3D Printing of Embedded Optical Elements for Interactive Devices. In Proceedings of the 25th Annual ACM Symposium on User Interface Software and Technology (UIST'12). ACM, New York, NY, USA, 589-598. DOI : http://dx.doi.org/10.1145/2380116.2380190 